\title{
The multifaceted use of Lactobacillus reuteri DSM 17938 in a pediatric clinic: a retrospective observational study
}

\author{
Dhanasekhar Kesavelu*
}

Consultant Paediatric Gastroenterologist, SS Childcare and Apollo Childrens Hospital, Chennai, India

Received: 01 July 2021

Accepted: 29 July 2021

*Correspondence:

Dr. Dhanasekhar Kesavelu,

Email: drdskgastro@gmail.com

Copyright: $\odot$ the author(s), publisher and licensee Medip Academy. This is an open-access article distributed under the terms of the Creative Commons Attribution Non-Commercial License, which permits unrestricted non-commercial use, distribution, and reproduction in any medium, provided the original work is properly cited.

\begin{abstract}
Background: Lactobacillus reuteri DSM 17938 is a well-studied probiotic which colonizes many mammals. In humans, $L$. reuteri is found in various body sites including the gastrointestinal tract, urinary tract, skin and breast milk. It fulfills all the prerequisites of being a good probiotic as described by the world gastroenterology organization.

Methods: This was a retrospective observational study, where a nutritional supplement $L$. reuteri DSM 17938 was given to 197 children, aged 0-16 years, along with normal diet. The participants were having complains of diarrhea, stomach pain and frequent hospitalization. Various parameters were recorded such as sex, age, anthropometry-weight and height (every visit), diagnosis, reason for outpatient visits and various demography including but not limited tobirthplace, current location, year in school, reason for hospital admission (if any). Paired t test was used to find difference in weight and height between each visit.

Results: A total of 118 subjects were included in the analysis. Five drops of nutritional supplement $L$. reuteri DSM 17938 was given. Supplementation of $L$. reuteri reduced the need for hospital admissions. $96 \%$ children did not require hospital admission. It improved the overall health of the children. There was a statistical significance in the height and weight between first and second visits $(\mathrm{p}<0.001)$. A $7.65 \%$ weight increase was noted in between first and second visit. A $2.32 \%$ height increase was seen between first and second visit.

Conclusions: L. reuteri DSM 17938 given as a nutritional supplement improved the health of the children and reduced the need for hospital admissions.
\end{abstract}

Keywords: Nutritional supplements, Height, Weight, Children, L. reuteri DSM17938, Probiotic

\section{INTRODUCTION}

Malnutrition in children may directly correlate with stunted growth, impaired cognitive development and reduced academic performance. It causes the impairment of cell-mediated immunity through its impact on phagocytic and cytokine activities. Fermented foods or probiotics have been known to modulate the gut microflora stimulating an immune response. ${ }^{1}$ Probiotics are defined as live organisms, which when administered in adequate dosages help to incur a health benefit for the host. ${ }^{2}$
Probiotics improve the child's growth by preventing infections and micronutrient deficiencies by increasing the absorption of certain nutrients such as calcium, zinc and vitamin B12. ${ }^{3,4}$ By improving gut health, probiotics also reduce the incidence of diarrhea. ${ }^{3,4}$ Overall, the benefits of probiotics in children with specific disease conditions such as acute infectious diarrhea, antibiotic-associated diarrhea, necrotizing enterocolitis in very low birth weight infants, childhood atopy, Helicobacter pylori infection and infantile colic have been established. ${ }^{5-8}$ 
L. reuteri was first isolated in 1962 and has been characterized as a heterofermentative species that grows in oxygen-limited atmospheres. ${ }^{9}$ It confers desired probiotic properties as it colonizes the gastrointestinal (GI) tract of humans and animals. ${ }^{9}$ L. reuteri can withstand a wide variety of $\mathrm{pH}$ environments by employing multiple mechanisms that allow it to successfully inhibit pathogenic microorganisms. By reducing antimicrobial intermediaries, $L$. reuteri has also been found to reduce the incidence of infections in children. ${ }^{9,10}$ Pertaining to these benefits, the use of $L$. reuteri as a nutritional supplement in infants and children has been supported by several guidelines including European Food Safety Agency (EFSA) and European Society of Paediatric Gastroenterology Hepatology and Nutrition (ESPGHAN). ${ }^{11,12}$ Previous research on Lactobacillus strains have shown that they help in the prevention and treatment of numerous GI tract disorders including enteric infections, antibiotic-associated diarrhea, necrotizing enterocolitis in preterm neonates, inflammatory bowel disease, colorectal cancer and irritable bowel syndrome. ${ }^{10}$ Current available medical evidence supports it in the prevention/management of multiple pathological conditions in children.

Since the indigenous environment of the human GI tract is suitable for its growth, L. reuteri has shown several generalized health benefits like reduction of infections, improvement in feed tolerance, enhancement of nutrient absorption, modulation of host immune responses, promotion of gut mucosal integrity, reduction of bacterial translocation and overall health promotion. ${ }^{13}$ Due to its ability to survive in low $\mathrm{pH}$ and enzyme-enriched environments, it survives in the proximal end of the digestive tract where it adheres to the epithelium for hostprobiotic interaction, competing with pathogenic microorganisms. ${ }^{14}$ This is another mechanism through which, the administration of $L$. reuteri helped in reducing the incidence of infections in pediatric hosts. ${ }^{13}$

An increased use of probiotics over the last few decades has primarily been because of its role in the management of acute diarrheal disease. ${ }^{15}$ However, probiotics offer serve several other benefits in infants and children including an improvement in anthropometry (height and weight parameters). ${ }^{4}$ While the role of $L$. reuteri in reducing diarrhea has been widely studied, this study aimed to analyze the overall health benefits of probiotics in children with a focus on the use of L. reuteri.

The manuscript aimed to determine the effect of $L$. reuteri on improvements in the GI diseases, its effects on the need for hospitalization and the effect on height and weight of the children, in order to determine its direct benefit with respect to growth and development in children.

\section{METHODS}

\section{Participants}

For this retrospective observational analysis, data was collected from 197 children who visited our clinic, SS childcare, Chennai, India. The participants were within the age group of 0-16 years and having complains of diarrhea, stomach pain and frequent hospitalizations.

\section{Intervention}

All participants of this study had been prescribed with $L$. reuteri DSM 17938 to study its impact on reduction in the events of hospitalization as well as change in height and weight parameters. All the participants of the study were treated equally and data retrospectively analyzed for one year. There were no treatment/control groups for the purpose of comparison and no randomization sequence was followed.

The use of $L$. reuteri as a nutritional supplement had been formulated for Indian children as per the ICMR guidelines. Its use was indicated as a probiotic food supplement containing the patented lactic acid bacterium, L. reuteri DSM 17938.

For our study, this nutritional supplement was administered to all the participants as OD (once daily)/BD (twice daily) formulations depending on their independent clinical profile. Five drops orally each were administered with the help of a plastic dropper along with promotion of intake of a normal diet. No lifestyle changes were recommended to the participants. They were followed up at regular intervals to determine any change in their symptoms.

\section{Data collection}

Demographic parameters such as their sex, age and educational status were recorded along with necessary anthropometric data including the weight and height (in $\mathrm{kgs}$ and $\mathrm{cms}$ ) at every visit. Their primary diagnosis, reason for outpatient visits, reason for hospital admission as well reasons and frequency of second visits were recorded along with their overall symptoms during visits.

\section{Statistical analysis}

Following data collection, SPSS software was used for statistical analysis. Paired t test was used to analyze the difference between height and weight between the visits. The $\mathrm{p}$ value $<0.05$ was considered statistically significant. Paired t test and Wilcoxon signed rank test were used to find the difference between the weights between the visits.

\section{Ethical considerations}

Since the participants were below 18 years of age, parents/guardians provided written informed consent before entering the study. The participants of the study were not followed up for their consent and only those agreeing to the purpose of the study were included based on their written permissions. 


\section{RESULTS}

\section{Demographic results}

A total of 197 children were initially selected on the basis of our retrospective analysis, but 79 dropped out. Thus, data from 118 children was considered. The mean age of the participants was $2.89 \pm 3.3$ years and the gender distribution included 64 boys and 54 girls. Most of the participants of the study were currently residing in Chennai $111(94 \%)$ and were Indian. The study included data from a diverse group of children from different socioeconomic strata. $100 \%$ children received 5 oral drops of the nutritional supplement, $98 \%$ received it once a day (OD) and only $1.7 \%$ got it twice a day (BD). Mode of delivery at parturition was normal vaginal delivery for 37 (31.4\%) children and lower (uterine) segment caesarean section (LSCS) for $81(68.6 \%)$ children (Table 1). Data regarding their gestational age at delivery was not recorded. All the participants of the study had vaccinations up to date according to the Indian academy of pediatrics recommendation.

\section{Primary diagnosis of the participants}

Mean age of children on the date of commencement of the nutritional supplement was $2.44 \pm 3.03$ years. After the primary diagnosis, $21(17.8 \%)$ children were diagnosed with functional abdominal pain, 23 (19.5\%) for prophylaxis of infections, $12(10.2 \%)$ had recurrent infections, $14(11.9 \%)$ with recurrent URTI, $11(9.3 \%)$ with functional constipation, $1(0.8 \%)$ child for each was diagnosed with infantile regurgitation, exaggerated gastrocolic reflex, infantile dyschezia and small intestinal bacterial overgrowth (Figure 1 and 2).

\section{Relationship between L. reuteri and symptom reduction in the participants}

After a follow up period of 12 weeks, it was observed that one $(0.8 \%)$ child was diagnosed for each pathological conditions including aerophagia, antibiotic associated diarrhea, functional abdominal pain, functional constipation and functional constipation, functional diarrhea, GERD, infantile dyschezia. However, 61 (51.7\%) out of 118 children were well (Figure 3).

\section{Impact of $L$. reuteri on the rate of hospital admission}

Administration of $L$. reuteri helped in reducing the need for hospital admissions. With respect to hospital admissions, it was observed that after the first visit by 118 participants, 64 participants $(54.2 \%)$ did not visit again (no visit). 16 participants $(13.6 \%)$ came for a second hospital visit owing to their clinical symptoms after $L$. reuteri administration. Only one $(0.8 \%)$ child came for a third visit (Figure 4$)$.

Table 1: Demographic data.

\begin{tabular}{|ll|}
\hline Parameters & Values \\
\hline Age (mean \pm SD) (in years) & $2.89 \pm 3.3$ years (range 0-16) \\
\hline Gender & \\
\hline Male & 64 \\
\hline Female & 54 \\
\hline Mode of delivery & $37(31.4)$ \\
\hline Normal (N, \%) & $81(68.6)$ \\
\hline LSCS (N, \%) & 100 \\
\hline Dosing & 98.3 \\
\hline 5 drops (\%) & 1.7 \\
\hline OD dose $(\%)$ & \\
\hline BD dose (\%) & \\
\hline
\end{tabular}

Table 2: Mean and standard deviation of weight during 1st and 2nd hospital visits.

\begin{tabular}{|lllll|}
\hline Pair 1 & Mean & N & Standard deviation & Standard error mean \\
\hline 1st visit weight & 12.252 & 118 & 7.6813 & .7071 \\
\hline 2nd visit weight & 13.190 & 118 & 8.1072 & .7463 \\
\hline
\end{tabular}

Table 3: Mean and standard deviation of height during 1st and 2nd hospital visits.

\begin{tabular}{|lllll|}
\hline Pair 1 & Mean & N & Standard deviation & Standard error mean \\
\hline 1st visit height & 84.43 & 118 & 26.802 & 2.467 \\
\hline 2nd visit height diagnosis & 86.39 & 118 & 26.523 & 2.442 \\
\hline
\end{tabular}




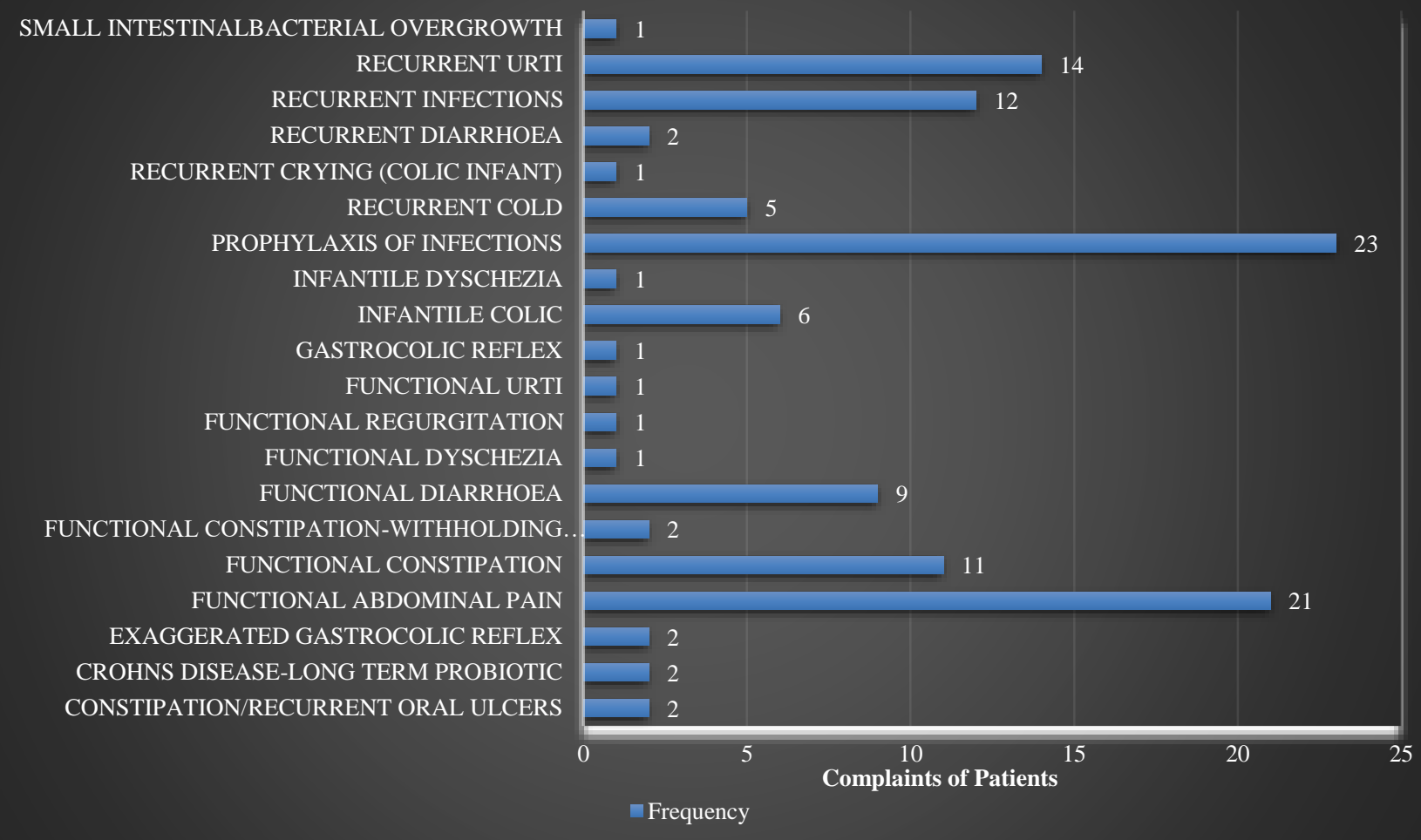

Figure 1: Frequency of primary complaints of the patient at the first hospital visit (prior to the L. reuteri administration).

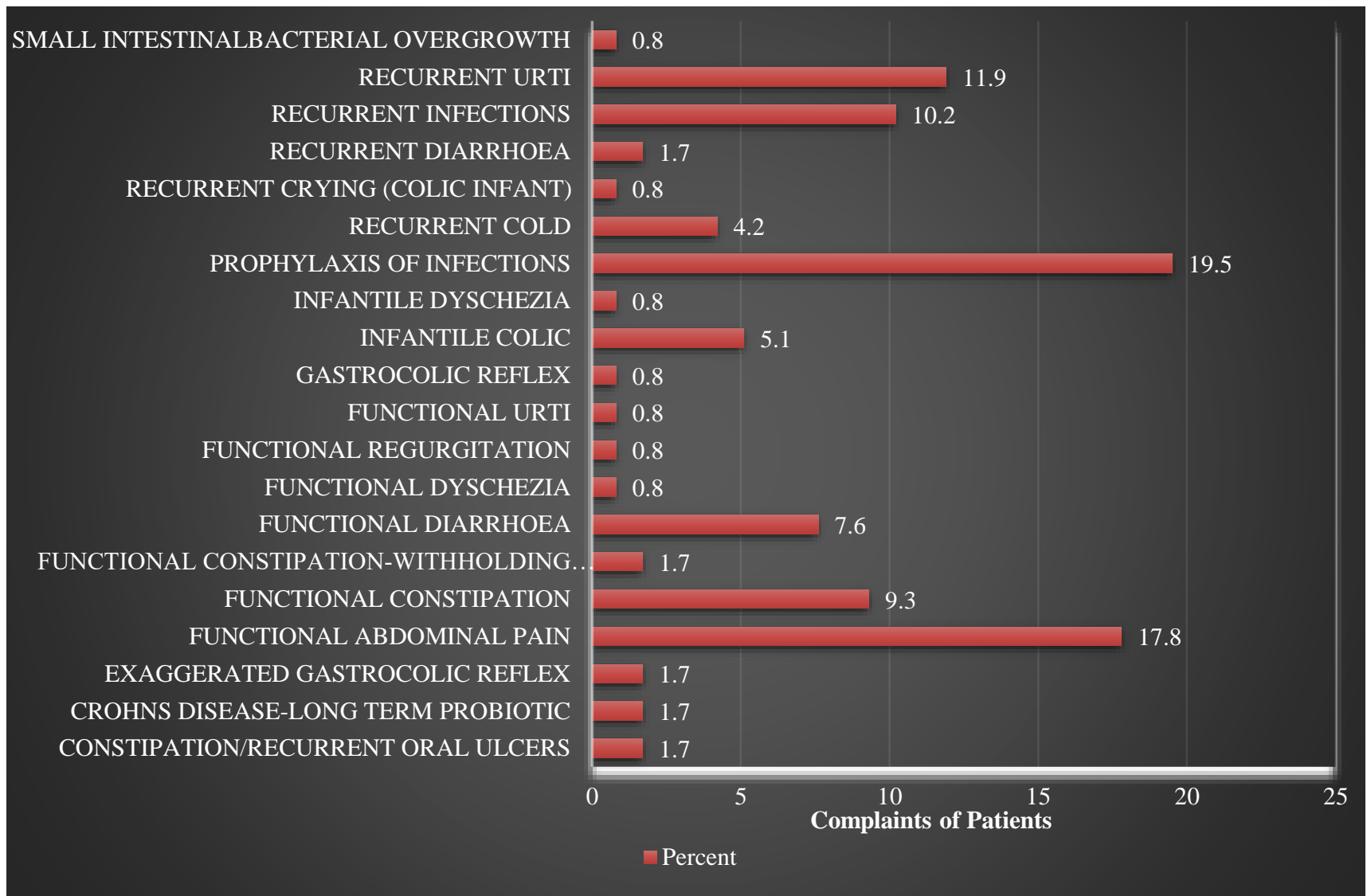

Figure 2: Percentage of primary complaints of the patient at the first hospital visit (prior to the $L$. reuteri administration). 


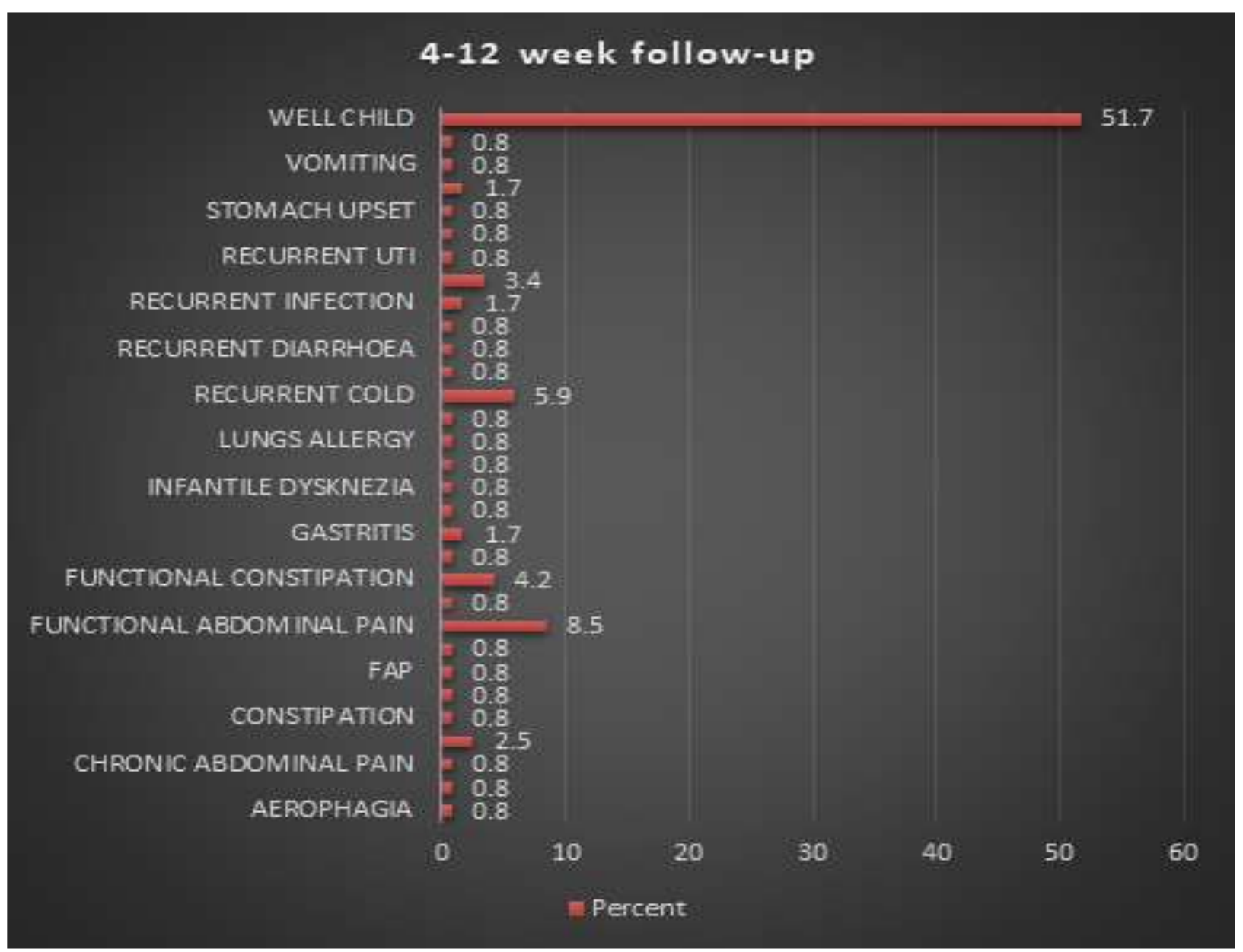

Figure 3: Patient complaints after a follow up period of 4 to 12 weeks after L. reuteri administration.

\section{Number of Hospital Visits (\%)

$$
(\mathbf{n}=118)
$$

60

50

40

30

20

10

0

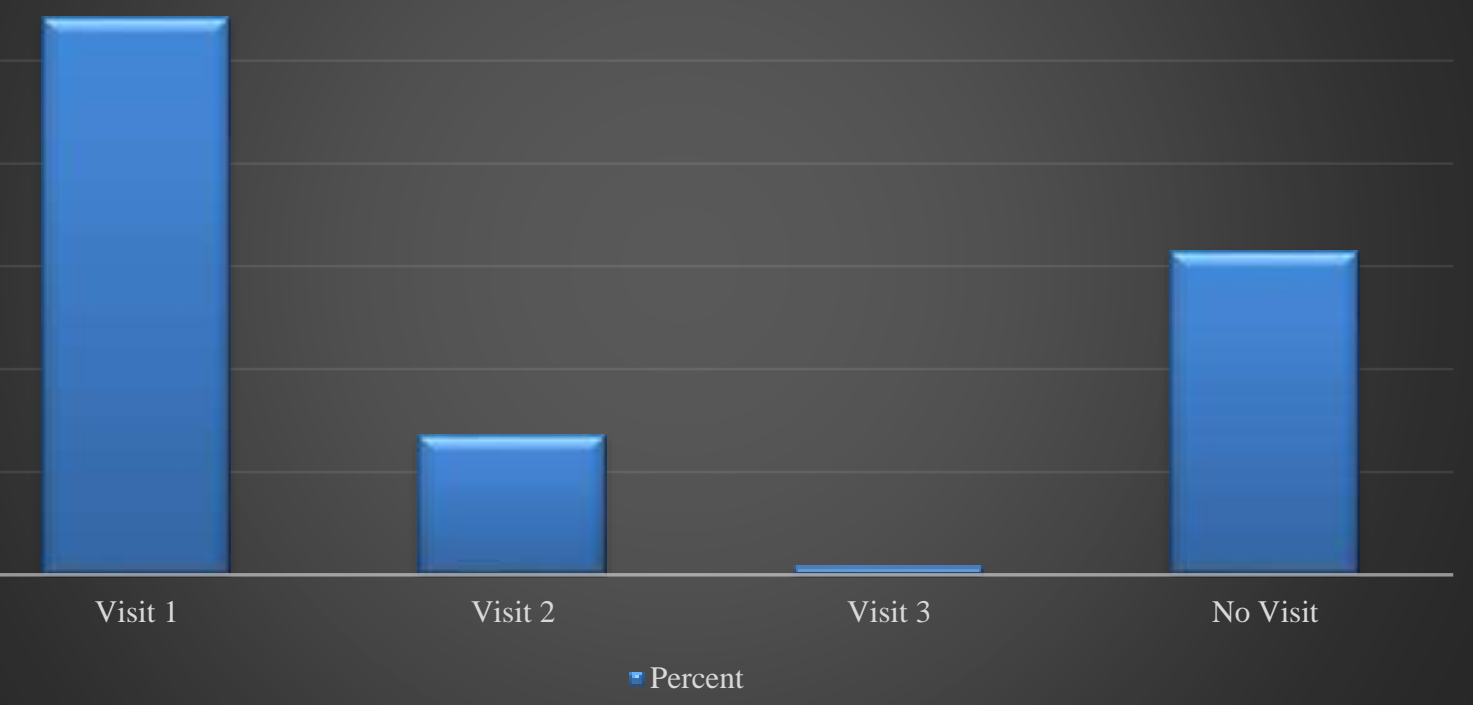

Figure 4: Number of hospital visits of the participants (first visit by 118 participants was the initial visit before the administration of $L$. reuteri). 


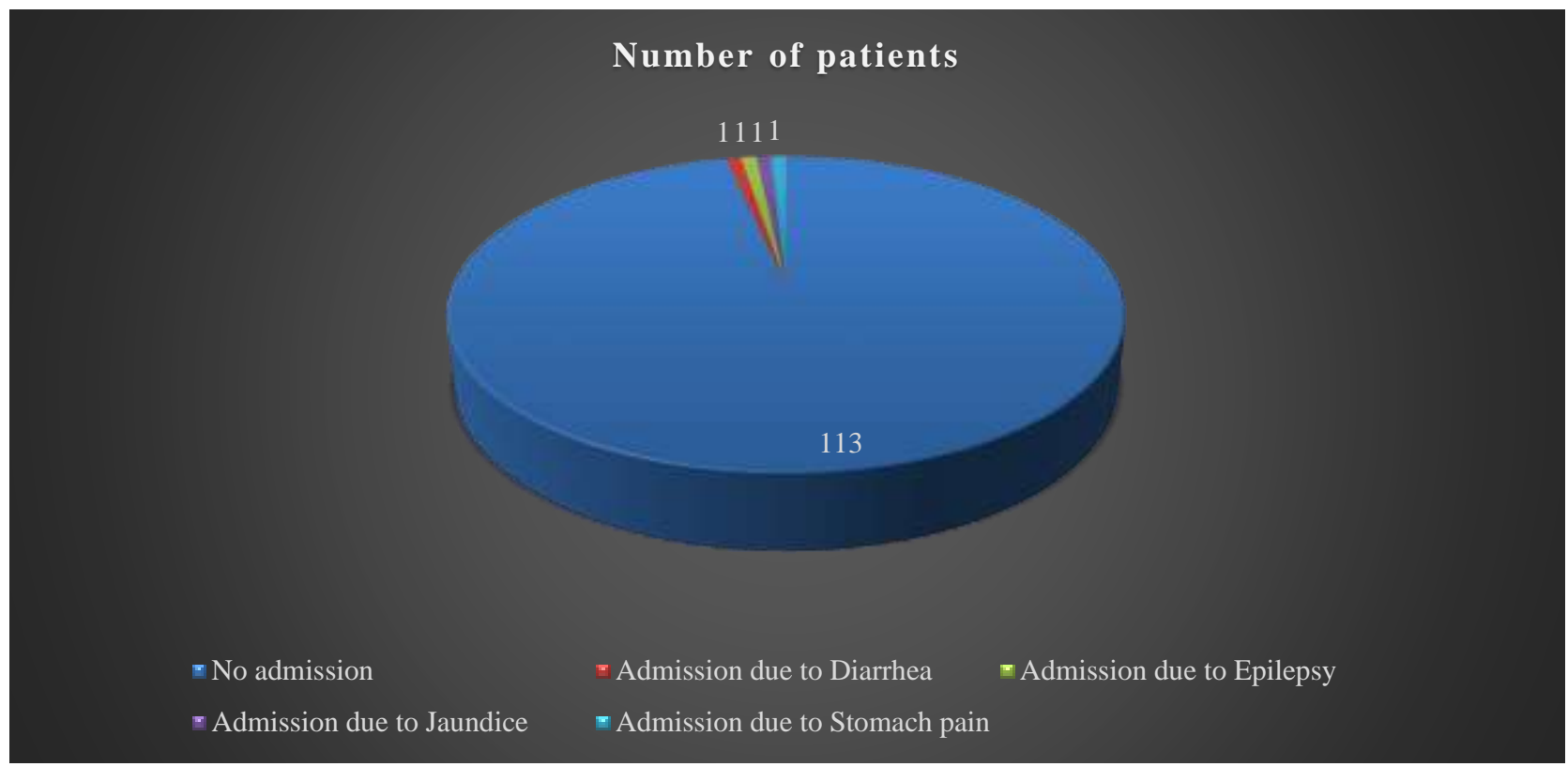

Figure 5: Reason of hospital admission of the participants.

Among participants reporting for the second time, 45 participants $(38.1 \%)$ were well at the time of their presentation to the clinic. 28 participants $(23.7 \%)$ reported due to URTI, 14 participants $(11.9 \%)$ came for a general visit and nine participants $(7.6 \%)$ came for routine vaccination.

113 out of 118 participants (95.8\%) did not require hospital admission during our study (Figure 5). Of the five children who required inpatient admission, abdominal pain, diarrhea, seizures, sepsis and jaundice were the respective diagnosis, which were observed owing to their individual clinical profile and were not considered as side effects of L. reuteri administration.

\section{Impact of L. reuteri administration on height, weight and growth parameters of the participants}

The intake of $L$. reuteri significantly improved the anthropometric parameters of the participants. The mean weight during first visit was $12.252 \pm 7.6 \mathrm{~kg}$ and during second visit was $13.19 \pm 8.1 \mathrm{~kg}(\mathrm{p}<0.001)$ (Table 2). $7.65 \%$ increase in weight was observed at the second visit. Statistical differences in the height of the participants were observed at the second visit $(\mathrm{p}<0.001)$. The mean height during first visit was $84.43 \pm 26.8 \mathrm{~cm}$ and during second visit was $86.39 \pm 26.5 \mathrm{~cm}$ (Table 3 ).

\section{DISCUSSION}

The results of the study indicated that administration of $L$. reuteri improved patient symptoms and their overall status of health, which was reflected by reduced need for hospital admissions and significantly improved the anthropometric development of children, after a follow up of 12 weeks.
Improvement in clinical symptoms similar to our study had also been highlighted in the literature. A multi-center RCT (randomized controlled trials) $(\mathrm{N}=127)$ depicted that the use of $L$. reuteri alongside the standard treatment protocol of the patient helped in reducing the duration of hospital stay because of improvement of GI symptoms. ${ }^{16}$ Its use also helped in improving major symptoms of the patient at day one itself as indicated by a meta-analysis report of three RCTs. ${ }^{17}$ Thus, the administration of $L$. reuteri in children helped in reducing diarrheal symptoms and prevented their recurrence.

In a 2012 RCT of 74 children, the use of $L$. reuteri significantly reduced the duration of diarrhea when compared with placebo. ${ }^{18}$ Along with this, it also reduced the risk of recurrence of symptoms and worsening of symptoms in the form of watery diarrhea. ${ }^{18}$ Further, $L$. reuteri supplementation also reduced the incidence of infectious diarrhea as indicated by a 2015 trial by Dinleyici et al $(\mathrm{N}=64) .{ }^{19}$

A majority of patients in our study presented with functional abdominal pain $(21.7 \%)$. Thus, overall reduction in hospitalization visits in our study also suggested that $L$. reuteri supplementation favored a reduction functional abdominal pain. This was similar to the findings of a 2010 trial of 60 pediatric patients where L. reuteri supplementation over a four week follow up period was shown to reduce pain intensity in patients with functional abdominal pain. ${ }^{20}$ The improvement in patient symptoms as presented in Figure 2, following the use of $L$. reuteri can be attributed to the anti-inflammatory and antimicrobial actions of $L$. reuteri. Supplementation of $L$. reuteri decreased the microbial translocation from the gut lumen to the tissues impeding the initiation of 
inflammation and facilitating the amelioration of inflammatory conditions. ${ }^{9}$ An RCT study of 117 infants supported the safe use of $L$. reuteri in infants with colic disease including those with neutropenia. In these infants, resolution of symptoms was noted within a period of three weeks. ${ }^{21}$ Literature evidence also pointed out that $L$. reuteri can help in reducing the incidence of diarrhea while having a suitable efficacy for the treatment of functional constipation. ${ }^{22}$ Similar study supported the use of $L$. reuteri as a nutrient supplement added to cow's milk. ${ }^{22}$ This was also helpful in reducing the incidence of diarrhea in malnourished children. ${ }^{23}$ In preterm infants, on the other hand, the use of $L$. reuteri had evidenced a reduction in food intolerances as supported by a double blinded RCT of 94 neonates. ${ }^{24}$ Since $L$. reuteri facilitated reduction in most common primary diagnosis of the participants enrolled in our study, $51.7 \%$ of participants of our study were symptom free at the time of their subsequent hospital visit. Thus, L. reuteri also helped in managing diarrhea, inflammatory conditions, neutropenia, functional abdominal pain and functional constipation.

In this study, administration of $L$. reuteri had positive impact on height and weight. The average increase in height was $1.96 \mathrm{~cm}$ during the study period while the average increase in weight was $0.938 \mathrm{~kg}(\mathrm{p}<0.001)$. Similar results have been found in the literature, which emphasized the role of $L$. reuteri in improving height and weight status of children. In six months RCT study of 494 participants within the age group of one to six years, it was found that L. reuteri caused significantly higher weight gain and more favorable growth velocities. ${ }^{23}$ In this study, L. reuteri was found to be more beneficial than $L$. casei, which only improved weight and not height. ${ }^{23}$ Systematic reviews and meta-analysis conducted in developing countries have also supported that probiotic supplementation helped in reducing growth status in undernourished children. ${ }^{15}$

Based on our observations, we recommend the use of $L$. reuteri as a nutritional supplement in children in the age group of 0 to 16 years to enhance their overall growth and development and five drops of once daily formulations can be safely prescribed in children who frequently present to the hospital with GI symptoms alongside their normal meal. Its use in healthy term infants had been supported by an RCT pediatric participants conducted in South East Asia, which concluded that its use helped in achieving WHO growth parameters. ${ }^{25}$

Since our study only included participants with clinical complaints who presented to our hospital, the evidence was insufficient to support the routine use of $L$. reuteri in infants and children. Although no side effects were associated with the use of $L$. reuteri in our study or in the literature, in children with severe symptoms, close follow up was recommended along with continuation of their medical management since a direct impact of $L$. reuteri in the improvement of existing medical conditions had not yet been concluded..$^{22,26}$ In critically ill children and children with immunocompromised patients, cautionary use was recommended. ${ }^{27}$

\section{Limitations}

One of the primary limitations of our study was the small sample size of the research and the use of a retrospective research design. Further, our study was a single-centre study and there was no comparison between the intervention and control arms.

\section{CONCLUSION}

The findings of our retrospective analysis of 118 children (mean age $2.89 \pm 3.3$ years) convincingly concludes that the use of $L$. reuteri as a probiotic or a nutrient supplement helps in reducing the rate of hospital admissions and overall hospital visits among children as observed in this COHORT. This is because it causes an improvement in patient symptoms, which can be attributed to its nutritional and immunomodulative effects. It also facilitates significant improvement in height and weight parameters. Throughout the course of our study, no major side effects pertaining to the use of $L$. reuteri were reported. Thus, it can be safely recommended in both infants and children who frequently report to the outpatients because of gastrointestinal symptoms like diarrhea or functional abdominal pain. The administration of $L$. reuteri has been found to be particularly beneficial in reducing the risk of recurrence of diarrheal symptoms and pain reduction in cases of functional abdominal pain or functional constipation. For healthy preterm infants, its use has been associated with an improvement in food tolerance. Since most of the included studies for our evaluation had a small sample size and were single-centre studies, it is recommended that the routine prescription of $L$. reuteri in infants/children as well as in critically ill patient be determined based on a large, multi-centre randomized clinical trial.

\section{Recommendations}

Although our research provided useful insights into the role of $L$. reuteri in the growth and development of children, $t$ test could not be run for symptom improvement and hospital admission, so, their statistical significance could not be determined. Thus, future research must be conducted in the form of a multi-centre randomized controlled trial where successful t-tests are conducted for each result so that the drawbacks of the present research can be met. This research must also include healthy participants so that insights into regular supplementation of L. reuteri in the pediatric population can be obtained.

\section{ACKNOWLEDGEMENTS}

Authors would like to thank Dr. Punit Srivastava and Dr. Nidhi Gupta for the medical writing and editorial support in the preparation of this article. 
Funding: No funding source

Conflict of interest: None declared

Ethical approval: The study was approved by the Institutional Ethics Committee

\section{REFERENCES}

1. Solis B, Samartin S, Gomez S, Nova E, Rosa BDL, Marcos A. Probiotics as a help in children suffering from malnutrition and diarrhoea. Eur J Clin Nutr. 2002;56(3):S57-9.

2. Food and Agricultural Organisation-World Health Organisation. Joint FAO/WHO Expert Consultation. 2001. Evaluation of Health and Nutritional Properties of Probiotics in food including Powder Milk with Live Lactic Acid Bacteria. Available at: http://www.fao. org/3/a0512e/a0512e.pdf. Accessed on 3 June 2021.

3. He M, Yang YX, Han H, Men JH, Bian LH, Wang GD. Effects of yogurt supplementation on the growth of preschool children in Beijing suburbs. Biomed Environ Sci. 2005;18(3):192-7.

4. Scholz-Ahrens KE, Ade P, Marten B, Weber P, Timm W, Açil Y, et al. Prebiotics, probiotics, and synbiotics affect mineral absorption, bone mineral content, and bone structure. J Nutr. 2007;137:838-46.

5. Allen SJ, Martinez EG, Gregorio GV, Dans LF. Probiotics for treating acute infectious diarrhoea. Cochrane Database Syst Rev. 2010;2010(11):003048.

6. Szajewska H, Kotowska M, Mrukowicz JZ, Arma M, Mikolajczyk W. Efficacy of Lactobacillus GG in prevention of nosocomial diarrhea in infants. J Pediatr. 2001;138(3):361-5.

7. AlFaleh K, Anabrees J. Probiotics for prevention of necrotizing enterocolitis in preterm infants. Evid Based Child Health. 2014;9(3):584-671.

8. Thomas DW, Greer FR. American Academy of Pediatrics Committee on Nutrition, American Academy of Pediatrics Section on Gastroenterology H. Nutrition. Probiotics and prebiotics in pediatrics. Pediatrics. 2010;126(6):1217-31.

9. $\mathrm{Mu} \mathrm{Q}$, Tavella VJ, Luo XM. Role of Lactobacillus reuteri in human health and diseases. Front Microbiol. 2018;9:757.

10. Lebeer S, Vanderleyden J, Keersmaecker SCD. Genes and molecules of lactobacilli supporting probiotic action. Microbiol Mol Biol Rev. 2008;72(4):728-64.

11. Szajewska H, Guarino A, Hojsak I, Indrio F, Kolacek $\mathrm{S}$, Shamir R, et al. Use of probiotics for management of acute gastroenteritis: a position paper by the ESPGHAN working group for probiotics and prebiotics. J Pediatr Gastroenterol Nutr. 2014;58(4):531-9.

12. EFSA Panel on Dietetic Products, Nutrition and Allergies (NDA). Scientific opinion on the substantiation of health claims related to Lactobacillus reuteri ATCC 55730 and decreasing potentially pathogenic intestinal microorganisms (ID 904) pursuant to article 13(1) of regulation (EC) No 1924/2006. EFSA J. 2009;7(9):1243.
13. Tubelius P, Stan V, Zachrisson A. Increasing workplace healthiness with the probiotic Lactobacillus reuteri: a randomised, double-blind placebocontrolled study. Environ Health. 2005;4:25.

14. Krumbeck JA, Marsteller NL, Frese SA, Peterson DA, Ramer-Tait AE, Hutkins RW, et.al. Characterization of the ecological role of genes mediating acid resistance in Lactobacillus reuteri during colonization of the gastrointestinal tract. Environ Microbiol. 2016;18(7):2172-84 .

15. Onubi OJ, Poobalan AS, Dineen B, Marais D, McNeill G. Effects of probiotics on child growth: a systematic review. J Health Popul Nutr. 2015;34:8.

16. Dinleyici EC, PROBAGE Study Group, Vandenplas Y. Lactobacillus reuteri DSM 17938 effectively reduces the duration of acute diarrhoea in hospitalised children. Acta Paediatr. 2014;103(7):300-5.

17. Urbańska M, Gieruszczak-Białek D, Szajewska H. Systematic review with meta-analysis: Lactobacillus reuteri DSM 17938 for diarrhoeal diseases in children. Aliment Pharmacol Ther. 2016;43(10):1025-34.

18. Francavilla R, Lionetti E, Castellaneta S, Ciruzzi F, Indrio F, Masciale A, et al. Randomised clinical trial: Lactobacillus reuteri DSM 17938 vs placebo in children with acute diarrhoea-a double-blind study. Aliment Pharmacol Ther. 2012;36(4):363-9.

19. Dinleyici EC, Dalgic N, Guven S, Metin O, Yasa O, Kurugol Z, et al. Lactobacillus reuteri DSM 17938 shortens acute infectious diarrhea in a pediatric outpatient setting. J Pediatr (Rio J). 2015;91(4):392-6.

20. Romano C, Ferrau V, Cavataio F, Iacono G, Spina M, Lionetti $\mathrm{E}$, et al. Lactobacillus reuteri in children with functional abdominal pain (FAP). J Paediatr Child Health. 2014;50(10):68-71.

21. Fatheree NY, Liu Y, Taylor CM, Hoang TK, Cai C, Rahbar MH, et al. Lactobacillus reuteri for infants with colic: a double-blind, placebo-controlled, randomized clinical trial. J Pediatr. 2017;191:170-8.

22. Urbańska M, Szajewska $H$. The efficacy of Lactobacillus reuteri DSM 17938 in infants and children: a review of the current evidence. Eur J Pediatr. 2014;173(10):1327-37.

23. Agustina R, Bovee-Oudenhoven IM, Lukito W, Fahmida U, Rest OVD, Zimmermann MB, et al. Probiotics Lactobacillus reuteri DSM 17938 and Lactobacillus casei CRL 431 modestly increase growth, but not iron and zinc status, among Indonesian children aged 1-6 years. J Nutr. 2013;143(7):1184-93.

24. Kaban RK, Hegar B, Rohsiswatmo R, Rohsiswatmo $\mathrm{R}$, Handryastuti S, Amelia N, et al. Lactobacillus reuteri DSM 17938 improves feeding intolerance in preterm infants. Pediatr Gastroenterol Hepatol Nutr. 2019;22(6):545-53.

25. Bharani R, Biswas A, Lee J, Tran LA, Pecquet S, Steenhout P. Normal growth of infants receiving an infant formula containing Lactobacillus reuteri, galacto-oligosaccharides, and fructo-oligosaccharide: a randomized controlled trial. Matern Health Neonatol Perinatol. 2015;1:9. 
26. Hojsak I. Probiotics in children: what is the evidence? Pediatr Gastroenterol Hepatol Nutr. 2017;20(3):13946.

27. Hojsak I, Fabiano V, Pop TL, Goulet O, Zuccotti GV, Çokuğraş FC, et.al. Guidance on the use of probiotics in clinical practice in children with selected clinical conditions and in specific vulnerable groups. Acta Paediatr. 2018;107(6):927-37.
Cite this article as: Kesavelu D. The multifaceted use of Lactobacillus reuteri DSM 17938 in a pediatric clinic: a retrospective observational study. Int J Basic Clin Pharmacol 2021;10:1134-42. 